\title{
MODULAÇÃO COMPORTAMENTAL E ADESTRAMENTO BÁSICO DE CÃES DO CENTRO DE CONTROLE DE ZOONOSES DE ARAUCÁRIA - PR
}

\author{
(Behavioral modulation and basic training of dogs from the zoonosis control center of \\ Araucaria - PR)
}

\section{Louise Bousfield de Lorenzi Tezza, Flávia de Mello Wolff, Feliphe Boaventura, Carla Forte Maiolino Molento ${ }^{1}$ \\ ${ }^{1}$ Correspondência: carlamolento@ufpr.br}

RESUMO: O objetivo foi colaborar para um avanço no conhecimento a respeito do impacto positivo de intervenções etológicas em cães de Centros de Controle de Zoonoses. Foi realizada avaliação comportamental em 25 cães do CCZ do município de Araucária/PR, atividade que auxiliou na identificação do manejo adequado para cada cão, assim como na definição de estratégias para redução de comportamentos indesejáveis. Houve redução importante de latidos excessivos, pulos nas pessoas, tração da guia e agressividade direcionada a pessoas e a outros cães. Os animais foram adestrados exclusivamente com reforços positivos, sendo que os comandos "aqui" e "senta" foram aprendidos por 25 animais (100\%). Dar a pata foi ensinado a 16 animais (64\%) e 13 animais (52\%) aprenderam a deitar. Ao final foi possível observar melhorias no bem-estar dos animais, aumento de comportamentos desejáveis e redução daqueles inadequados. Tais resultados motivam pesquisas futuras sobre o impacto do manejo no sucesso das adoções.

Palavras-chave: bem-estar animal; comportamento; interação ser humano-animal

ABSTRACT: The objective was to collaborate to advances in knowledge about the positive impact of ethological interventions in dogs from municipal shelters. A behavioral assessment was performed with 25 dogs from the Zoonosis Control Center of Araucária, Paraná, Brazil, which aided the identification of appropriate management for each one and for strategies to reduce undesirable behaviors. There was an important reduction of excessive barking, jumping on people, pulling while in leash and aggression toward people and other dogs. The animals were trained exclusively with positive reinforcement. The commands "come" and "sit" were learned by 25 animals (100\%). Give paw was taught to 16 animals (64\%) and 13 animals $(52 \%)$ learned to "lie down". It was possible to observe improvements in animal welfare, an increase in desirable behaviors and a reduction in inappropriate ones. These results motivate further research concerning the impact of handling on the adoption success.

Key Words: animal welfare, behavior, human-animal interaction 


\section{INTRODUÇÃO}

A manutenção da saúde humana de qualquer região inclui $\mathrm{o}$ combate às zoonoses, o qual por sua vez apresenta como um de seus objetivos o controle de populações animais que compartilham doenças com 0 ser humano. O cão, quando presente nas ruas em desacordo com as diretrizes da guarda responsável, está sujeito a riscos para seu bem-estar e também oferece desafios para 0 bem-estar humano, podendo causar agravos à sociedade, como mordeduras e transmissão de doenças (Dalla Villa, 2010).

Em virtude do avanço e divulgação da ciência de bem-estar animal (Molento e Calderón, 2009), da existência de legislação ambiental (Lei Federal 9.605/98) que apoia a proteção dos animais e da comprovada ineficiência de métodos de extermínio com o objetivo de controle de populações caninas (WHO, 2005), o direcionamento das atividades dos CCZs brasileiros vêm mudando. Ao invés da eliminação dos animais, o foco atualmente está em fomentar a guarda responsável entre a população.

Sabe-se que uma das principais justificativas para 0 abandono de animais na rua ou em abrigos passa pela afirmação de que 0 animal apresenta comportamentos indesejáveis (Scarlett, 1999; New, 2000; Salman et al., 2000; Lord et al., 2008). Essa também é a maior causa de retorno de animais recém-adotados às entidades ou abrigos (Mondelli e Previde, 2004; Shore et al., 2005). Adestrar os animais pode ser uma maneira de reduzir tais causas de abandono. Tal medida pode estar relacionada à prevenção e atenuação de alterações comportamentais dos animais, como agressividade para com pessoas e outros animais, hiperatividade, vocalização, timidez ou medo excessivos, entre outras que constam como mais relatadas de acordo com Salman et al. (2000) e Shore et al. (2005).

O objetivo deste trabalho foi colaborar para um avanço no conhecimento a respeito do perfil comportamental de cães recolhidos das ruas e do impacto positivo de intervenções etológicas em cães de CCZs sobre o bem-estar dos animais e diminuição de comportamentos indesejáveis.

\section{MATERIAL E MÉTODOS}

O estudo foi realizado no $\mathrm{CCZ}$ do município de Araucária, Paraná, durante os meses de setembro e outubro de 2010. Participaram do trabalho 25 cães saudáveis, sendo 11 machos e 14 fêmeas, todos jovens acima de quatro meses ou adultos. Os animais foram recolhidos das ruas de acordo com o programa de recolhimento seletivo (agressivos, doentes ou machucados) e estavam aguardando adoção. Inicialmente foi criada uma forma de avaliar os animais quanto ao comportamento, com base em literatura existente sobre o assunto (Borg et al., 1991; Hsu e Serpell, 2003; De palma et al., 2005; Segurson et al., 2005; Taylor e Mills, 2006). A avaliação foi composta por 10 testes simples e foi realizada em 20 dos 25 animais do CCZ que participaram do estudo, sendo que cinco cães foram adotados antes da avaliação ou eram filhotes abaixo dos seis meses de idade. Ela permitiu avaliar de maneira objetiva o comportamento do animal em cinco quesitos, necessitando pouco material ou instalações quando comparado com outras análises (Netto e Planta, 1997). Os quesitos avaliados foram: receptividade a pessoas; dominância e agressividade a pessoas e a outros animais; excitabilidade e confiança do animal. Uma mão artificial foi confeccionada para avaliação de animais suspeitos de serem agressivos. A avaliação foi realizada com o uso de escala numérica pré-definida de zero a 
dez e os resultados foram divididos em cinco faixas, duas superiores (característica muito observada e observada), duas inferiores (característica pouco observada e quase ausente) e a quinta como "característica não observada". A média dos resultados numéricos obtidos serviu para classificar as características individuais de cada animal, assim como para definir um perfil para os 20 animais trabalhados (Tab. 1). A partir do resultado destes testes foram geradas recomendações específicas para a adoção de cada cão e traçado um perfil comportamental dos animais.

Os animais foram adestrados quanto aos comandos básicos "aqui", "senta", "dá a pata" e "deita" exclusivamente com o uso de estímulos positivos, como elogios, carinho e petiscos. O uso de "clicker" ocorreu ocasionalmente. Para modificar comportamentos indesejáveis dos animais, inicialmente foi ensinado um comportamento alternativo ou foram fornecidas condições para que este fosse realizado. Estímulos positivos foram utilizados para a modulação. Apenas no caso de agressão a outros cães e pular nas pessoas ocorreu uso ocasional do estímulo aversivo de aspersão de água. A comparação das situações antes e depois da intervenção foi realizada com a descrição dos comportamentos observados e com 0 uso de fotografias e filmagens.

\section{RESULTADOS E DISCUSSÃO}

Os 20 animais avaliados apresentaram de forma geral uma resposta bastante positiva quanto à receptividade a pessoas, que se deve provavelmente ao bom manejo em que são mantidos no CCZ de Araucária. O escore médio dos cães neste quesito foi de $8,23 \pm 1,84$ sendo que $90 \%$ (18 animais) se encaixaram nas duas faixas superiores, apresentando grande interesse por contato humano e interação, como observado na Tab. 1. Animais com estas características comportamentais tendem a permanecer na parte da frente do canil, o que aumenta a taxa de adoção segundo Thorn et al. (2006).

Quanto ao grau de dominância em relação às pessoas, 60\% (12 animais) dos cães avaliados foram classificados como levemente submissos; quatro animais se situaram nas faixas extremas, dois como muito dominantes e dois como muito submissos. É interessante notar que três dos animais observados nas faixas extremas apresentaram algum grau de agressividade durante 0 teste. Dos 20 animais avaliados, $14 \quad(70 \%)$ não apresentaram qualquer grau de agressividade relacionada a pessoas, ficando o escore geral em $0,36 \pm 0,84$ (Tab. 1). Observou-se que $67 \%$ dos cães enquadrados nas faixas "dominante" e "muito dominante" demonstraram agressividade, contribuindo com a hipótese de relação entre estas características. Já nas faixas "submisso" e "muito submisso", a incidência de agressividade foi de $14,3 \%$. Essa relação, embora reconhecida por alguns autores (Line e Voith, 1986; Hillestad, 2001), é contestada por outros (Yin, 2007; Bradshaw et al., 2009); sendo que os resultados aqui obtidos apresentam coerência com os primeiros.

Com relação à dominância em relação $a$ outros cães a média ficou em 6,3 $\pm 2,0$, sendo que um quarto (5/20) dos animais apresentou características muito dominantes e metade (10/20) apresentou tendências dominantes durante a avaliação. Já com relação à agressividade a média foi de 0,75 \pm 1,41 , sendo que dos 20 cães avaliados, seis animais $(30 \%)$ demonstraram agressividade direcionada a outros cães. 


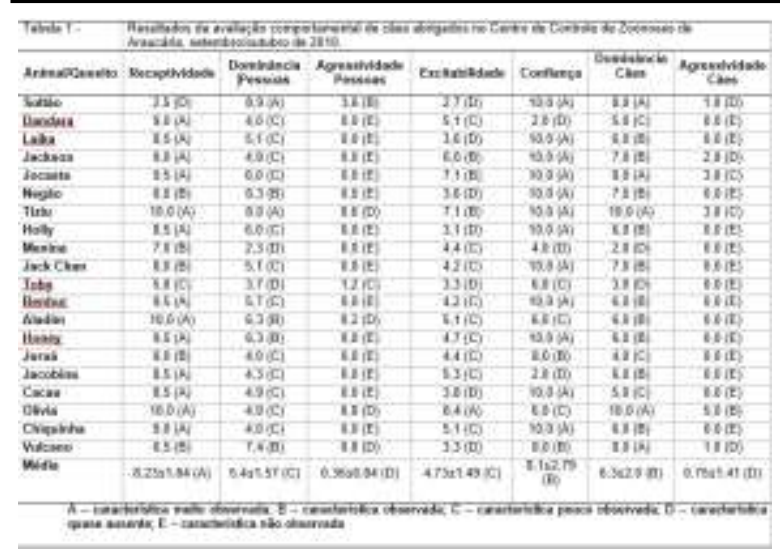

Quando o grau de excitabilidade foi avaliado, percebeu-se que $80 \%(16 / 20)$ dos animais demonstraram-se tranquilos ou muito tranquilos. O escore geral para esta característica foi de $4,73 \pm 1,49$. No quesito confiança a média dos animais avaliados ficou em $8,1 \pm 2,79$. A maioria dos animais $(70 \%)$ se mostrou confiante ou muito confiante.

Os resultados de cada animal durante a avaliação comportamental permitiram montar um perfil do cão e tal conhecimento auxiliou tanto na modulação comportamental - nos casos em que foi verificado algum comportamento indesejável, como também na seleção do melhor perfil de adotante para aquele animal. A recomendação entregue aos adotantes com instruções referentes ao manejo inicial do animal no novo ambiente, também se basearam nos resultados da avaliação. Essas recomendações sobre como evitar problemas comportamentais podem ajudar a garantir uma adoção bem sucedida (Herron et al., 2007).

Os comportamentos indesejáveis observados no grupo dos 25 animais foram latidos excessivos, com incidência de $60 \%$; pulos nas pessoas $(80 \%)$, agressividade contra pessoas $(20 \%)$, agressividade contra outros cães (28\%), automutilação por lambedura $(4 \%)$, timidez excessiva com as pessoas (4\%) e não acompanhar a pessoa no passeio com guia frouxa (36\%).

Após intervenção, foi observada uma redução importante nos latidos. No início, a entrada de uma pessoa no Bloco de Controle Animal fazia com que a maior parte dos cães ganisse ou latisse, mesmo se a pessoa fosse conhecida. $O$ diagnóstico etológico revelou que desta forma os animais conseguiam atenção. Os funcionários do CCZ foram orientados a não recompensar os animais com reforços positivos no momento em que apresentassem comportamentos indesejados, assim como foram orientados a reforçar comportamentos adequados. Após a modulação, o número de cães que vocalizavam de forma insistente para os adotantes passou de $60 \%$ a $12 \%$, e algumas vezes foi possível mostrar todos os cães sem um único latido. $A$ interação $e$ recompensa em momentos de calma e silêncio ensinaram aos animais que esta é a forma mais eficaz de conseguir atenção uma vez que latir não trazia resultados. $O$ adestramento para os animais sentarem e deitarem também auxiliou, pois os animais passaram a realizar esses comportamentos espontaneamente como alternativa à vocalização e pular em busca de atenção.

O comportamento de pular nas pessoas é prejudicial porque suja as roupas e pode machucar ou assustar as pessoas, principalmente em cães de grande porte. No início do trabalho foi observado que $80 \%$ dos animais pulavam na maioria das vezes ao encontrar uma pessoa. Após a modulação, essa taxa caiu para $28 \%$, o que equivale dizer que 7 de 25 cães continuaram pulando nas pessoas na maioria das vezes. O resultado não foi ainda mais positivo porque alguns animais foram adotados rapidamente, antes do que seria necessário para alterar o comportamento, bem como as pessoas que tinham contato com os cães continuaram a recompensar positivamente com carinho cães de pequeno porte que pulavam, por não 
verem esse comportamento como indesejável.

A agressividade direcionada a pessoas foi observada em cinco animais de forma rotineira. Dos cinco, quatro animais aparentemente apresentavam agressividade por dominância e um por medo, sendo bastante submisso. Dos quatro por dominância, dois não apresentaram mais comportamentos agressivos após a modulação ("Tiziu", "Aladim" - Tabela 1). Os outros dois ("Vulcano", "Sultão" - Tabela 1) foram adotados antes da modulação completa, mas o treinamento aliado ao adestramento básico permitiu uma aproximação segura com os possíveis adotantes. Estes dois cães obedeceram a comandos, foram recompensados permitindo o contato físico com as pessoas, que gostaram dos animais e os adotaram.

No caso do animal agressivo submisso ("Toby" - Tabela 1), a estratégia adotada foi diferente. $O$ animal não permitia qualquer aproximação sem se mostrar agressivo, rosnando e mostrando os dentes, porém mantinha uma postura submissa, conforme pode ser conferido na Figura. 1. Esse comportamento era exibido para todas as pessoas, com exceção dos dois tratadores, incluindo aproximação com oferta de petiscos, que 0 animal recusava. Tal modulação foi realizada com associação lenta e gradual de pessoas a resultados positivos, basicamente alimento, por meio de muitas repetições rápidas por dia. Sempre recompensando o animal com alimento, antes que o mesmo apresentasse comportamento agressivo, foram ultrapassadas diversas etapas: primeiramente, era jogado o alimento para o canil do "Toby", sendo que a pessoa continuava seu caminho, sem falar ou olhar para o animal. Aos poucos, a pessoa passou gradativamente a se aproximar cada vez mais, falar, manter contato visual, abrir a gaiola, encostar no animal e posteriormente acariciá-lo por todo o corpo antes de dar o petisco. Além disso, passou-se a adestrá-lo com comandos básicos. Após o mesmo adaptar-se à manipulação e carícias, estar à vontade fora do canil e obedecer a comandos, o animal passou a permanecer solto com cães dóceis e a interação com outras pessoas foi incentivada. Esta atitude teve por objetivo generalizar a associação de pessoas a reforços positivos. Após a modulação, o animal, que antes não permitia contato e esboçava invariavelmente alto grau de agressividade, passou a se mostrar mais receptivo (Figura 1) e permitir 0 contato de pessoas conhecidas sem reforço e desconhecidas com 0 fornecimento de petiscos. Pode-se concluir que dos cinco animais, em dois a intervenção apresentou sucesso completo e em três houve melhora importante.

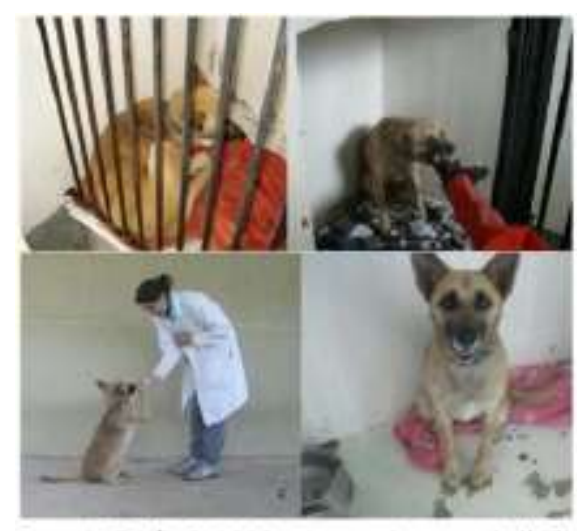

Figura 1 -Toby'; cilo nicialmante agrossivo por medo (A, B) agrondeu comandos basicos (C) e se tomou docl e receptivo Ás pessoas apús a modaçaco (D)

Sete animais demonstraram comportamento de agressão direcionado a outros cães. Entretanto, foi realizada intervenção em três, por uma questão de limitação de tempo. A agressividade direcionada a animais foi observada em duas situações, sendo que a primeira ocorria apenas em caso de oferta de alimento ou atenção quando havia outros cães por perto. Após modulação, dois animais ("Tiziu", 
"Aladim" - Tabela 1) que anteriormente exibiam esse comportamento deixaram de se mostrar agressivos nessas situações. A outra situação envolveu dois animais que quando em contato visual mostravam-se agitados, rosnavam e latiam intensamente. Para estes foram realizadas sessões de aproximação com obediência a comandos e reforço positivo quando permaneciam calmos. Ao final das duas sessões de treinamentos, ambos os animais se mantiveram tranquilos e obedeceram a comandos sem parecer se importar com a presença do outro cão (Figura 2). Houve progresso importante, porém seriam necessárias mais sessões de treinamento para que eles se comportassem da mesma forma espontaneamente. Das quatro situações em que ocorreu agressividade contra outros cães e houve intervenção, duas foram resolvidas e em duas houve progresso importante.

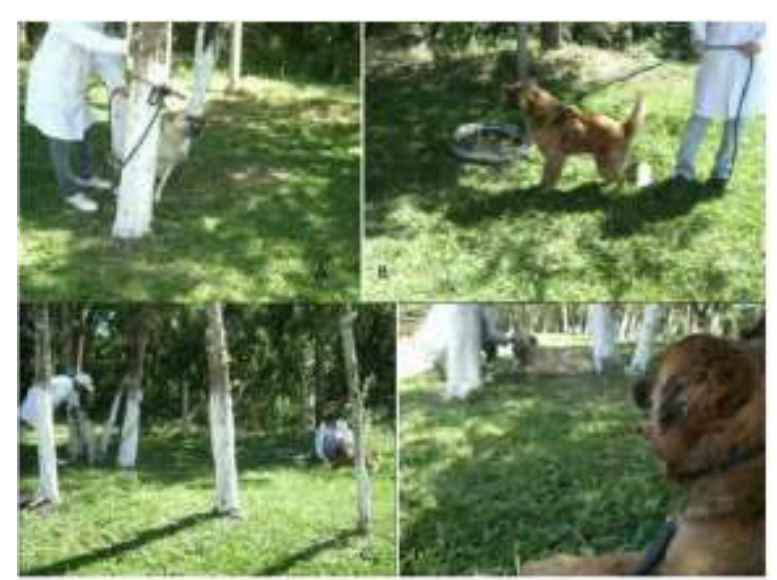

Figura 2 - "Ben-Hur' e "Tiziur, dois cảes machos que apresentavam agressividado com a presencat do outro (A, B), depoes do alguns minulos praticando comandos bésicos e recebendo recompensas (C), se apresentaram tranquilos (D).

Uma cadela chamada "Menina" (Tabela 1) apresentava lesão provocada por lambedura, na região dorsal da cauda, estando a ferida em processo de cicatrização há mais de um ano. Outras técnicas haviam sido empregadas, como uso de colar elisabetano e uso de substâncias amargas, sem resultado. Passou-se a realizar enriquecimento ambiental para aumentar os estímulos, fornecimento de brinquedos para roer, adestramento para aumentar a confiança do animal - visto ser este inseguro e ansioso - e modulação visando objetivamente diminuir o medo e a timidez observada, por meio de associação de pessoas a estímulos positivos como alimentos. $O$ animal não permitia a interação física, porém acompanhava as pessoas e abanava a cauda, mantendo-se a curta distância. Por meio do registro de imagens (Figura 3), foi possível observar melhora da ferida, não linear, ao longo das quatro semanas de modulação. Entretanto, uma conclusão confiável exigiria acompanhamento mais prolongado. Após a intervenção, o animal passou a pedir atenção encostando e se aproximando com maior frequência das pessoas e a obedecer a comandos de pessoas que acabou de conhecer, mesmo sem qualquer recompensa alimentar.

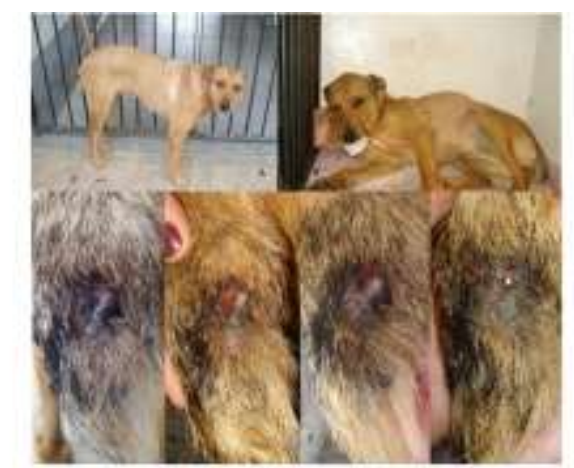

Figura 3 - Menina", cacela com comportamento assisatado e submissa (A) que, apos ser oferacido enriquecimento ambiental (B) * adestramento, apresentec meihoca em lesso auta infligida, Intorvalo de uma soenana ontra cadia imagem.

Outro comportamento indesejável identificado foi não caminhar adequadamente com a guia. Sete animais apresentavam inicialmente 0 comportamento de puxar a guia e dois filhotes não sabiam caminhar com a mesma. Dos nove, um foi adotado antes que fosse iniciada a modulação e dois reduziram bastante o comportamento, embora ainda puxassem ocasionalmente, sendo adotados antes do término da intervenção. Os outros seis tiveram o comportamento modulado 
de maneira muito efetiva, caminhando junto com a pessoa sem tracionar a guia. O comportamento de tracionar a guia pode causar tanto incômodo ao dono que este decide não mais levar o animal para passeios, reduzindo o grau de bem-estar dos cães.

É importante notar algumas particularidades do CCZ de Araucária que influenciaram os resultados obtidos. Mesmo com grande número de cães existentes no local durante o período, que nunca foi inferior a 30, a preocupação com o bem-estar dos animais sempre existiu, havendo um interesse em manter os animais em liberdade e socializados com pessoas e outros cães. A segunda particularidade é a grande rotatividade dos animais, ocorrendo, portanto oscilações na quantidade de cães que apresentavam os comportamentos indesejados ao longo do estágio. Novos cães que apresentassem comportamentos indesejáveis como latir excessivamente, estimulavam outros cães a aumentarem os latidos, até que os primeiros aprendessem o comportamento alternativo. A adoção dos animais antes da alteração completa do comportamento indesejável ocorreu, porém progressos importantes foram obtidos. Recomendações ao adotante sobre como prevenir a volta do comportamento não-desejável ou como continuar a modulação para diminuir a ocorrência deste, de forma segura e com reforços positivos, foram efetuadas pelo CCZ de Araucária.

Os animais foram adestrados com os comandos básicos: "aqui", "senta", "deita" e "dá a pata". Ao todo, 25 animais participaram de sessões de treinamento. Foram realizadas 154 sessões, com média de 6,2 $\pm 3,7$ sessões por animal. A duração usual de cada sessão foi de 15 a 25 minutos. Devido ao tempo de permanência variável dos animais no CCZ de Araucária e às diferenças individuais, nem todos os animais aprenderam os quatro comandos. Dos 13 animais que permaneceram durante todo o período, 10 aprenderam todos os comandos. Dos três que não aprenderam, dois faziam uso diário de medicação para epilepsia. Um destes animais aprendeu dois comandos e o outro três. Quanto ao terceiro animal, não foi ensinado o "deita" porque ele já era excessivamente submisso e tímido (Menina - Tabela 1) e exibia este comportamento constantemente, não sendo desejável, pois a timidez excessiva é motivo de abandono e devolução de animais adotados (Salman et al., 2000, Shore et al. 2005).

Do total de 25 animais adestrados, apenas 12 permaneceram no CCZ durante todo o período de intervenção. Dos 25, 12 (48\%) aprenderam todos os quatro comandos, cinco (20\%) aprenderam três comandos e oito (32\%) aprenderam dois comandos. Foi considerado como aprendizagem do comando o fato do animal obedecer $90 \%$ das vezes, ainda que sob circunstâncias específicas pelo uso de recompensa em comida ou sem a presença de muitos cães ao redor. Os comandos "aqui" e "senta" foram os primeiros a serem ensinados e foram aprendidos pelos 25 animais (100\%). Os demais comandos foram ensinados após os primeiros estarem sendo executados com facilidade, o que conferiu o aprendizado de "dar a pata" por 16 animais (64\%) e 13 animais (52\%) aprenderam a deitar.

$\mathrm{O}$ adestramento realizado com reforços positivos forneceu aos animais uma oportunidade de interação positiva com as pessoas (Figura 4), proporcionou aumento da sociabilidade e gerou estímulo mental importante. Observouse ainda que os animais diminuíram, em decorrência do adestramento, a frequência de comportamentos indesejáveis como latir e pular ao aprenderem comportamentos 


alternativos para serem
recompensados. A agressividade por dominância foi reduzida a partir do momento em que os cães obedeceram a comandos, realizando uma interação positiva com seres humanos. Percebeuse ainda que o comportamento timidez excessiva foi atenuado pelo adestramento e os animais se mostraram mais confiantes na interação com as pessoas e facilitou também a criação de vínculo entre cão e adotante, por promover a realização de uma atividade positiva para o cão e valorizada pelo adotante. O interesse do candidato a adotante aumentou nos momentos em que cães adestrados eram visualizados e dos 25 animais treinados 17 tiveram a adoção concretizada dentro do período dos dois meses de intervenção. Tais resultados sugerem a importância do aprendizado de comandos básicos no aumento da taxa de adoção dos animais.

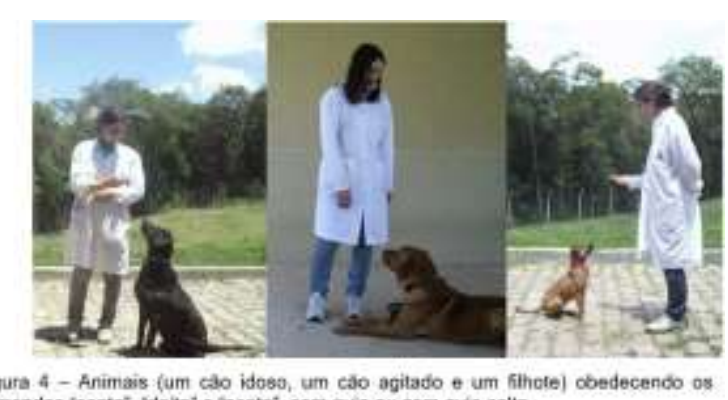

Figura 4 - Animais fum calo idoso, um cajo agitado e um fithote) obedecendo os comandos "senta', "deita'e 'senta", sem gua ou com quia solta.

A melhoria no bem-estar animal dos cães do CCZ de Araucária com a intervenção etológica foi percebida por meio de diagnóstico a partir das Cinco Liberdades do bem-estar animal (FAWC, 1993). Os animais reduziram comportamentos estereotipados como andar de um lado para o outro, pular nas grades e latir de forma crônica, que estão associados a baixo grau de bemestar. Os animais mostraram-se mais tranquilos e mais receptivos ao contato humano e com outros cães após o período, sugerindo aumento de sentimentos positivos. Estes estão associados a uma melhora na Liberdade
Psicológica do animal, que também é beneficiada pelo aumento de estímulos no ambiente, como enriquecimento ambiental. Este último também atua de maneira positiva na Liberdade Comportamental, pois permite ao animal exercer comportamentos naturais como buscar os alimentos e roer objetos, além do adestramento ter preenchido necessidades de interação decorrentes do comportamento social do cão doméstico.

O aumento da probabilidade do animal adestrado ter sentimentos positivos durante a atividade contribuiu para 0 aumento no bem-estar dos cães trabalhados. A oportunidade de estimular o cão social e mentalmente pode preencher necessidades associadas à sua saúde mental.

\section{CONCLUSÃO}

Ao final do período em que ocorreu adestramento e modulação comportamental, foi possível observar melhorias no bem-estar dos animais, aumento de comportamentos desejáveis e redução de comportamentos inadequados, o que pode aumentar a possibilidade de adoção e de sucesso pós-adoção dos animais. A avaliação comportamental inicial permitiu uma melhor abordagem para cada cão e recomendações mais efetivas ao adotante. Outras experiências similares devem ser realizadas e estudadas, visto os benefícios obtidos no CCZ de Araucária.

\section{AGRADECIMENTOS}

À toda a equipe do Centro de Controle de Zoonoses de Araucária, que muito contribuiu para a realização deste trabalho.

Aos Professores Doutores Tilde Rodrigues Froes e Alexander Welker Biondo, pelas proveitosas sugestões e importante apoio ao compor a banca examinadora durante a apresentação do TCC que deu origem a este trabalho. 


\section{REFERÊNCIAS}

BORG, J.V.D.; NETTO, W.; PLANTA, D. Behavioural testing of dogs in animal shelters to predict problem behavior. Journal of Applied Animal Welfare Science, v.32, issue2, p.237-251, 1991.

BRADSHAW, S.W.S.; BLACKWELL, E.J.; CASEY, R.A. Dominance in domestic dogs: useful construct or bad habit? Journal of Veterinary Behavior, v.4, p.135-144, 2009.

DALLA VILLA, P.; KAHN, S.; STUARDO, L. et al. Free-roaming dog control among OIE-member countries. Preventive Veterinary Medicine, v.97, p.58-63, 2010.

DE PALMA, C.; VIGGIANO, E.; BARILLARI, E.et al. Evaluating the temperament in shelter dogs. Behaviour, v.142, p.9-10, 1307-1328, 2005.

FARM ANIMAL WELFARE COUNCIL FAWC. Second report on priorities for research and development in farm animal welfare. MAFF Tolworth, U.K., 1993.

HERRON, M.H.; LORD, L.K.; HILL, L.N. et al. Effects of preadoption counseling for owners on house-training success among dogs acquired from shelters. Journal of the American Veterinary Medical Association, v.231, p.558562, 2007.

www.peteducation.com. HILLESTAD, $\mathrm{K}$. Aggression due to dominance. Foster and Smith Veterinary Services Department, 2001. Disponível em: $<$ http://www.peteducation.com/article.cf $\mathrm{m} ? \mathrm{c}=2+2085 \&$ aid $=2415>$. Acessado em: 22 mar. 2012.

HSU, Y.; SERPELL, J.A. Development and validation of a questionnaire for measuring behavior and temperament traits in pet dogs. Journal of the American Veterinary Medical Association, v.223, p.1293-1300, 2003.
JONES, A.C.; GOSLING, S.D. Temperament and personality in dogs (Canis familiaris): a review and evaluation of past research. Applied Animal Behaviour Science, v.95, p.153, 2005

LINE, S.; VOITH, V.L. Dominance aggression of dogs towards people: behavior profile and response to treatment. Applied Animal Behaviour Science, v.16, p.77-83, 1986.

LORD, L.K.; REIDER, L.; MEGHAN, E. et al. Health and behavior problems in dogs and cats one week and one month after adoption from animal shelters. Journal of the American Veterinary Medical Association, v.233, p.17151722, 2008.

MOLENTO, C.F.M.; CALDERÓN, N. Essential directions for teaching animal welfare in South America. Revue scientifique et technique - International Office of Epizootics, v.28, p.617-625, 2009.

MONDELLI, F.; PREVIDE, E. The bond that never developed: adoption and relinquishment of dogs in a rescue shelter. Journal of Applied Animal Welfare Science, v.7, p.253-266, 2004.

NETTO, W.J.; PLANTA, D.J.U. Behavioural testing for aggression in the domestic dog. Applied Animal Behaviour Science, v.52, issue 3, p.243-263, 1997.

NEW JR, J.C. Characteristics of shelterrelinquished animals and their owners compared with animals and their owners in U.S. pet owning households. Journal of Applied Animal Welfare Science, v.3, p.179-201, 2000.

SALMAN, M.D. HUTCHISON, J.; RUCH-GALLIE, R. et al. Behavioral reasons for relinquishment of dogs and cats to 12 Shelters. Journal of Applied Animal Welfare Science, v.3, p.93-106, 2000. 
SCARLETT, J.M. Reasons for relinquishment of companion animals in U.S. animal shelters: selected health and personal issues. Journal of Applied Animal Welfare Science, v.2, p.41-57, 1999.

SEGURSON, S.A.; SERPELL, J.A.; HART, B.L. Evaluation of a behavioral assessment questionnaire for use in the characterization of behavioral problems of dogs relinquished to animal shelters. Journal of the American Veterinary Medical Association, v.227, p.1755-61, 2005.

SHORE, E.R. Returning a recently adopted companion animal: adopters' reasons for and reactions to the failed adoption experience. Journal of Applied Animal Welfare Science, v.8, p.187-198, 2005.

TAYLOR, K.D.; MILLS, D.S. The development and assessment of temperament tests for adult companion dogs. Journal of Veterinary Behavior, v.1, p.94-108, 2006.

THORN, J.M.; TEMPLETON, J.J.; WINKLE, K.M.M. et al. Conditioning shelter dogs to sit. Department of Biology. Knox College. Journal of Applied Animal Welfare Science, v.9, p.25-39, 2006.

WORLD HEALTH ORGANIZATION WHO. World Health Organization Expert Consultation on Rabies. WHO Technical Report Series, 931, First Report, 450 p, 2005.

YIN, S. Dominance versus leadership in dog training. University of California, Davis, USA. Compendium on Continuing Education for the Practising Veterinarian, v.29, p.414-7, 432, 2007. 